\title{
Impact of Body Mass Index (BMI) on Chemotherapy-associated Toxicity in Ovarian Cancer Patients. A Pooled Analysis of the North-Eastern German Society of Gynecological Oncology (NOGGO) Databank on 1,213 Patients
}

\author{
JACEK PRZEMYSLAW GRABOWSKI ${ }^{1,2}$, ROLF RICHTER ${ }^{1}$, HANNAH RITTMEISTER ${ }^{1,2}$, \\ RADOSLAV CHEKEROV $^{1,2}$, HANNAH WOOPEN ${ }^{1,2}$ and JALID SEHOULI ${ }^{1,2}$ \\ ${ }^{1}$ European Competence Center for Ovarian Cancer (EKZE), \\ Department of Gynecology, Campus Virchow Klinikum, Charité - University Medicine of Berlin, Berlin, Germany; \\ ${ }^{2}$ North Eastern German Society of Gynecologic Oncology (NOGGO), Berlin, Germany
}

\begin{abstract}
Background/Aim: Chemotherapy-associated toxicity is one of the limiting factors regarding treatment efficacy, patient outcome and quality of life in this collective. Underweight or obese patients represent a major group in which the therapy seems to be more challenging. The aim of this analysis was to evaluate the impact of BMI on the toxicity in patients undergoing chemotherapy. Patients and Methods: The data of three prospective phase II/III studies ('Tower', 'Topotecan phase III' and 'Hector') of the NorthEastern German Society of Gynecological Oncology including 1,213 patients with recurrent ovarian cancer were retrospectively analyzed. The study was performed using logistic regression and Cox regression analysis. Results: The median age at diagnosis was 59 years. Sixty-seven (5.5\%) patients had BMI $<20$ and 272 (22.4\%) patients had BMI $>30$. Preterm termination of the chemotherapy was associated with lower BMI $(p=0.017)$. Moreover, nonhematological toxicity grade III/IV was mainly observed in underweighted women as well $(p<0.001)$. Patients with higher BMI more often presented with grade III/IV anemia ( $p=0.019)$ and as a consequence required blood transfusions more frequently ( $p=0.005)$. The overweight group was also associated with a higher number of co-medications. However, no difference in survival regarding BMI was observed in our study. Conclusion: Fewer chemotherapy
\end{abstract}

Correspondence to: Dr. med. Jacek Grabowski, Department of Gynecology, European Competence, Center for Ovarian Cancer, Charité Comprehensive Cancer Center, Charité-University Medicine of Berlin, Augustenburger Platz 1,13353 Berlin, Germany. Tel: +49 30450664355, e-mail: jacek.grabowski@ charite.de

Key Words: Ovarian cancer, BMI, chemotherapy, toxicity, underweight, overweight. cycles and preterm discontinuation were more frequent in patients with lower BMI. Hematological toxicity and higher medication intake appeared more often in patients with higher BMI.

Ovarian cancer, with approximately 150,000 deaths annually worldwide, is the leading cause of death among gynecological cancers (1). The influence of higher body mass index (BMI) on the risk and prognosis of high-grade serous ovarian cancer remains controversial $(2,3)$. There are very limited data available on BMI-related toxicities regarding systemic therapy (4-8). Patients with abnormal BMI often present comorbidities including cardiovascular disease, decreased liver and/or kidney function and co-medication, which should be taken into consideration during the treatment planning process. The influence of comorbidities on the survival of ovarian cancer patients remains unclear (9). For some of these patients, the optimal/standard therapy is not applicable which might result in decreased outcome. Regarding our prior study on toxicity in ovarian cancer patients $(10,11)$, the aim of this study was further evaluation focused on the impact of BMI on grade III/IV toxicities. Furthermore, a potential influence of BMI on chemotherapy dose reduction and survival in recurrent ovarian cancer patients was evaluated.

\section{Patients and Methods}

This individual participant data meta-analysis was conducted with the data of three large phase II/III trials comparing chemotherapy regimens in recurrent ovarian cancer patients run by the NorthEastern German Society of Gynecological Oncology (NOGGO).

TOWER study - Topotecan weekly versus conventional 5-day schedule in patients with platinum-resistant ovarian cancer (12). This phase II trial compared two different topotecan regimens [(1) weekly administration of $4.0 \mathrm{mg} / \mathrm{m}^{2} /$ week applied on days 1,8 and 15 of a 
28-day cycle, versus (2) conventional administration of $1.25 \mathrm{mg} / \mathrm{m}^{2} / \mathrm{d}$ on five consecutive days of a 21-day cycle] in recurrent epithelial ovarian cancer patients.

Topotecan phase III study - nonplatinum topotecan combinations versus topotecan alone for recurrent ovarian cancer (13). Topotecan alone was compared to two different topotecan combinations [(1) topotecan $1.25 \mathrm{mg} / \mathrm{m} 2 / \mathrm{d}$ on days 1 to 5 every 3 weeks or topotecan $1.0 \mathrm{mg} / \mathrm{m}^{2} / \mathrm{d}$ on days 1 to 5 plus $50 \mathrm{mg}$ of oral etoposide on days 6 to 12 every 3 weeks or (3) topotecan $0.5 \mathrm{mg} / \mathrm{m}^{2} / \mathrm{d}$ on days 1 to 5 plus gemcitabine $800 \mathrm{mg} / \mathrm{m}^{2}$ on day 1 and $600 \mathrm{mg} / \mathrm{m}^{2}$ on day 8 every 3 weeks] in this phase III trial in recurrent ovarian cancer patients.

Topotecan plus Carboplatin versus standard therapy with paclitaxel plus carboplatin $(P C)$ or gemcitabine plus carboplatin $(G C)$ or carboplatin plus pegylated doxorubicin (PLDC): a randomized phase-III trial of the NOGGO-AGO-Germany-AGO Austria and GEICO-GCIG Intergroup study (HECTOR) (14). The Hector phase III study was conducted to compare the combination of topotecan and carboplatin to three different standard carboplatin containing combinations [topotecan $0.75 \mathrm{mg} / \mathrm{m}^{2} / \mathrm{d}$ on days 1 to 3 , and carboplatin Area under the curve (AUC) 5 on day 3 after topotecan, every 3 weeks versus (PC) paclitaxel $175 \mathrm{mg} / \mathrm{m}^{2} / \mathrm{d}$ on day 1 , and carboplatin AUC 5 on day 1, every 3 weeks, (GC) gemcitabine 1000 $\mathrm{mg} / \mathrm{m}^{2} / \mathrm{d}$ on day 1 and 8 , and carboplatin AUC 4 on day 1 , every 3 weeks and (PLDC) pegylated doxorubicin $30 \mathrm{mg} / \mathrm{m}^{2}$ on day 1 and carboplatin AUC 5 on day 1, every 4 weeks] in platinum-sensitive recurrent ovarian cancer (first or second relapse).

Inclusion criteria of all three trials were age $\geq 18$ years, sufficient renal, liver and bone marrow function as well as an ECOG performance status $\leq 2$ for the Hector and topotecan phase III trials. Patients with serious or uncontrolled medical condition were not eligible for participation. Toxicities and their grades were documented according to the National Cancer Institute - Common Toxicity Criteria. Original data of the three described trials were provided by the NOGGO working group "Ovarian Cancer".

Statistics. Patients were divided into four groups according to BMI: (1) underweight - BMI <20, (2) normal weight $-\mathrm{BMI}=20-25$, (3) overweight - BMI $>25-30$ and (4) obese - BMI $>30$. IBM $^{\circledR}$ SPSS $^{\circledR}$ Statistics 23 (SPSS Inc. an IBM Company, Chicago, IL, USA) was used for statistical analyses. A $p$-value $<0.05$ was considered a significant result. The chi-square test was used to compare the prevalence of comorbidities, co-medications, and toxicities between the different BMI groups. Trends of these outcomes for increasing BMI groups were evaluated with Kendall's tau b. Odds ratios were estimated by logistic regression analyses for toxicities, prior discontinuation of chemotherapy and necessity of dose reduction after adjusting for covariates. Kaplan-Meier was used to estimate median survival. Survival curves were compared by the log-rank test. After adjusting for covariates, hazard ratios were estimated for progression-free and overall survival with Cox regression analyses.

\section{Results}

A total of 1,213 patients registered in the meta-database of the three mentioned trials were evaluated in our study. The median age of patients was 59 years (range $=19-84$ years). The majority of patients were initially diagnosed with advanced ovarian cancer (86.7\% FIGO III and IV). First ovarian cancer recurrence was reported in $86.5 \%$ of the analyzed patients (Table I).

The median body mass index in our patient's group was $25.7 \mathrm{~kg} / \mathrm{m}^{2}$ (range $\left.=15.5-48.4 \mathrm{~kg} / \mathrm{m}^{2}\right)$. Among those, 67 patients had BMI $<20 \mathrm{~kg} / \mathrm{m}^{2}$ and 273 patients had BMI $>30 \mathrm{~kg} / \mathrm{m}^{2}$. There were no significant differences regarding BMI groups within the analyzed trials $(p=0.933)$, ECOG $(p=0.964)$, age $(p=0.187)$, recurrence number $(p=0.475)$, grading $(p=0.478)$ and FIGO stage $(p=0.180)$ (Table I).

We found BMI-associated differences in the number of applied chemotherapy cycles $(p=0.018)$. Patients with lower BMI received fewer chemotherapy cycles in comparison to those with higher BMI (Table II). Discontinuation of chemotherapy was also associated with lower BMI $(p=0.017)$ (Table II). However, we did not find any differences regarding dose reduction according to BMI group $(p=0.275)$ (Table II).

Underweight patients showed a rate of grade III/IV anemia of $10.3 \%$. This number increased with increasing BMI to $19.8 \%$ in obese patients. Fatigue occurred in $5.1 \%$ of the obese patients but did not occur at all in underweight patients ( $p=0.009$ and $p=0.019$ respectively). Consequently, an association was found between BMI and blood transfusion frequency. Patients with $\mathrm{BMI}>30 \mathrm{~kg} / \mathrm{m}^{2}$ required blood transfusions more often in comparison to women with $\mathrm{BMI}<30 \mathrm{~kg} / \mathrm{m}^{2}(p=0.003)$. However, in multivariate logistic regression analysis adjusting for the entered study, number of cycles, age, FIGO staging, grading, number of recurrences, the correlation of BMI with grade III/IV anemia and hematological toxicity did not reach statistical significance (Figure 1).

Non-hematological grade III/V toxicities were statistically more frequently observed in patients with higher BMI $(p<0.001)$ which was also shown in multivariate logistic regression analyses adjusting for the covariates named above (Figure 1). Obese patients developed more often grade III/IV pulmonary toxicity than normal weight patients $(p=0.030$; Figure 1).

The impact of BMI levels regarding co-medication was also analyzed in our study. Anti-hypertensive $(p<0.001)$, cardiovascular $(p=0.001)$ and diabetes medication $(p=0.001)$ were more frequently taken by obese patients.

Furthermore, the survival rates according to different BMI values were analyzed. The median overall survival (OS) was 19.7 months and progression-free survival (PFS) 8 months for the whole group. Differences in OS $(p=0.386)$ and PFS $(p=0.488)$ rates among different BMI groups were not found (Figures 2 and 3) in both univariate and multivariate Cox regression analyses.

\section{Discussion}

Our analysis based on the data from 1213 patients showed differences in applied chemotherapy cycles, preterm therapy 
Table I. Baseline patient characteristics among the study population stratified by BMI.

\begin{tabular}{|c|c|c|c|c|c|}
\hline & \multicolumn{4}{|c|}{ BMI } & \multirow{2}{*}{$p$-Value } \\
\hline & $<20 \mathrm{~kg} / \mathrm{m}^{2}$ & $20-25 \mathrm{~kg} / \mathrm{m}^{2}$ & $>25-30 \mathrm{~kg} / \mathrm{m}^{2}$ & $>30 \mathrm{~kg} / \mathrm{m}^{2}$ & \\
\hline Patient number $(\%)$ & $67(5.5)$ & $468(38.6)$ & $403(33.4)$ & $273(22.5)$ & \\
\hline Median age (years) (range) & $59(19-84)$ & & & & \\
\hline FIGO $(\%)$ & & & & & $p=0.180$ \\
\hline $\mathrm{I} / \mathrm{II}$ & $7(10.3)$ & $56(12.0)$ & 46 (11.5) & $43(15.9)$ & \\
\hline III/IV & $61(89.7)$ & $406(87.3)$ & $351(87.3)$ & $228(83.8)$ & \\
\hline Unknown & 0 & $3(0.6)$ & $5(1.2)$ & $1(0.4)$ & \\
\hline Grading $(\%)$ & & & & & $p=0.478$ \\
\hline High-grade & $59(86.8)$ & $412(88.0)$ & $364(90.3)$ & $238(87.2)$ & \\
\hline Low-grade & $1(1.5)$ & $19(4.1)$ & $14(3.5)$ & $12(4.4)$ & \\
\hline Unknown & $8(11.8)$ & $37(7.9)$ & $25(6.2)$ & $23(8.4)$ & \\
\hline Histology (\%) & & & & & $p=0.023$ \\
\hline Serous & $60(88.2)$ & $355(75.9)$ & $296(73.4)$ & $198(72.5)$ & \\
\hline Mucinous & $1(1.5)$ & $20(4.3)$ & $11(2.7)$ & $13(4.8)$ & \\
\hline Endometrioid & $2(2.9)$ & $34(7.3)$ & $34(8.4)$ & $16(5.9)$ & \\
\hline Other & $5(7.4)$ & $59(12.6)$ & $62(15.4)$ & $44(16.1)$ & \\
\hline Unknown & 0 & 0 & 0 & $2(0.7)$ & \\
\hline Recurrent disease (\%) & & & & & $p=0.475$ \\
\hline 1 st & $59(86.8)$ & $412(88.2)$ & $337(83.6)$ & $239(87.5)$ & \\
\hline 2nd & $9(13.2)$ & $55(11.8)$ & $66(16.4)$ & $34(12.5)$ & \\
\hline ECOG & & & & & $p=0.964$ \\
\hline 0 & $26(38.2)$ & $208(44.4)$ & $181(45.1)$ & $107(39.5)$ & \\
\hline 1 & $36(52.9)$ & $217(46.4)$ & $196(48.9)$ & $151(55.7)$ & \\
\hline 2 & $6(8.8)$ & $39(8.3)$ & $21(5.2)$ & $12(4.4)$ & \\
\hline 3 & 0 & 0 & $1(0.2)$ & 0 & \\
\hline Unknown & 0 & $4(0.9)$ & $2(0.5)$ & $1(0.4)$ & \\
\hline
\end{tabular}

Table II. BMI and number of chemotherapy cycles, therapy discontinuation and dose reduction.

\begin{tabular}{|c|c|c|c|c|c|}
\hline \multirow[b]{2}{*}{$<20 \mathrm{~kg} / \mathrm{m} 2$} & \multicolumn{4}{|c|}{ BMI } & \multirow[t]{2}{*}{$p$-Value } \\
\hline & $20-25 \mathrm{~kg} / \mathrm{m}^{2}$ & $>25-30 \mathrm{~kg} / \mathrm{m}^{2}$ & $>30 \mathrm{~kg} / \mathrm{m}^{2}$ & & \\
\hline Cycles number (\%) & & & & & $p=0.018$ \\
\hline$<3$ & $12(17.6)$ & $80(17.1)$ & $42(10.4)$ & $31(11.4)$ & \\
\hline $3-5$ & $19(27.9)$ & $109(23.3)$ & $89(22.1)$ & $61(22.3)$ & \\
\hline$\geq 6$ & $37(54.4)$ & $179(59.6)$ & $272(67.5)$ & $181(66.3)$ & \\
\hline Discontinuation (\%) & & & & & $p=0.017$ \\
\hline Yes & $33(48.5)$ & $196(41.9)$ & $137(34.0)$ & $100(36.6)$ & \\
\hline No & $35(51.5)$ & $272(58.1)$ & $266(66 \%)$ & $173(63.4)$ & \\
\hline Dose reduction (\%) & & & & & $p=0.275$ \\
\hline Yes & $8(12.3)$ & $102(22.8)$ & $82(21.0)$ & $44(16.5)$ & \\
\hline No & $57(87.7)$ & $346(77.2)$ & $309(79.0)$ & $222(83.5)$ & \\
\hline
\end{tabular}

discontinuation, and grade III/IV toxicities in relation to BMI values. However, no differences in progression-free and overall survival were observed.

Body weight has a prognostic value regarding postoperative outcome and survival in ovarian cancer patients (15). Sehouli et al. showed a correlation between preoperative malnutrition ( $\mathrm{NRS} \geq 3$ ) with surgical outcome $(p=0.014)$ as well as poorer overall survival $(p=0.001)$. Regarding the BMI influence on the toxicity during chemotherapy and outcome, the available data are limited (11). Many ovarian cancer patients are fragile and at high risk of malnutrition. The majority $(>60 \%)$ of patients in our study either had BMI below or above the normal values. Therefore, a nutritional screening prior to chemotherapy 


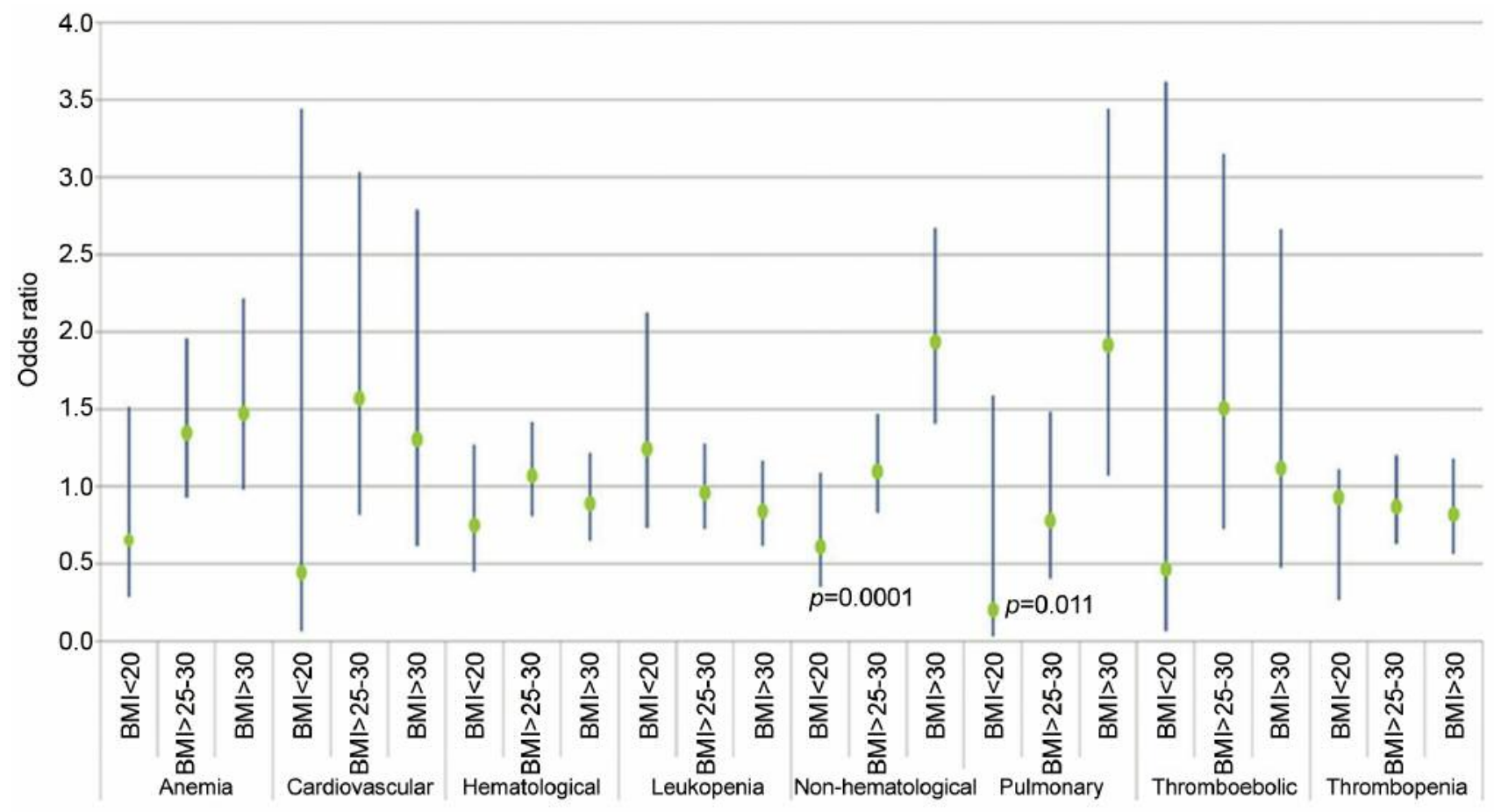

Figure 1. BMI and grade III/IV toxicities. Multivariate logistic regression analysis adjusting for entered study, number of cycles, ago, FIGO, grading, recurrence number. Reference value BMI $20-25 \mathrm{~kg} / \mathrm{m}^{2}$. Odds ratios illustrated as green dots, confidence intervals in blue.

should be mandatory. Moreover, the need for an individual and personalized clinical approach during systemic therapy in these patients seems to be needed. Nevertheless, no prospective studies were performed so far.

Associations between BMI, the number of applied chemotherapy cycles and prior therapy discontinuation in the studied cohort were found. Obese patients received more chemotherapy cycles and those with lower BMI discontinued therapy more frequently. However, no statistically significant dose reduction was observed between BMI subgroups. Dahlberg et al. analyzed the association between BMI and outcome of patients with advanced non-small cell lung cancer enrolled in Eastern Cooperative Oncology Group clinical trials (16). Obese patients more frequently discontinued treatment due to adverse events $(23.0 \%)$ when compared to those with lower BMI (16.8\%). In a regression model these results showed significant differences across BMI groups $(p=0.004)(16)$. These data stand in contrast to our observation which to our knowledge is the first study examining ovarian cancer patients.

Gutierrez et al. retrospectively analyzed hematological toxicity associated with carboplatin/paclitaxel chemotherapy in obese patients with gynecological cancer. Twenty-one overweight women were identified (BMI $\geq 27 \mathrm{~kg} / \mathrm{m}^{2}$ ). Among those the rate of grade III/IV anemia and thrombocytopenia was significantly higher in univariate analysis, $p<0.05$ and $p<0.002$ respectively (4). These patients more frequently required G-CSF and/or erythropoietin/blood transfusion (4). Our data show similar results regarding anemia and blood transfusion. An early infusion of erythropoietin, might lead to reduction of anemia and/or fatigue and therefore fewer interruptions/interval prolongations of the scheduled therapy in ovarian cancer patients with lower BMI. Regarding non-hematological toxicities our study showed an association with higher BMI. Dahlberg et al. did not find any differences in nonhematological toxicities regarding BMI groups in non-smallcell lung cancer patients (16).

Patients with higher BMI were found to take significantly more co-medications. Cardiovascular and diabetes medication were the most frequent among these patients. Recently, our working group showed that the more medication was taken by patients, the higher the risk of overall grade III/IV toxicities, hematological and non-hematological. However, no influence of polypharmacy on survival or prior discontinuation of therapy was reported (10). Nevertheless, due to association with grade III/IV toxicities, obese patients and those taking higher amount of co-medications should be thoroughly monitored during therapy. Further prospective trials on co-medication impact should be planned. 


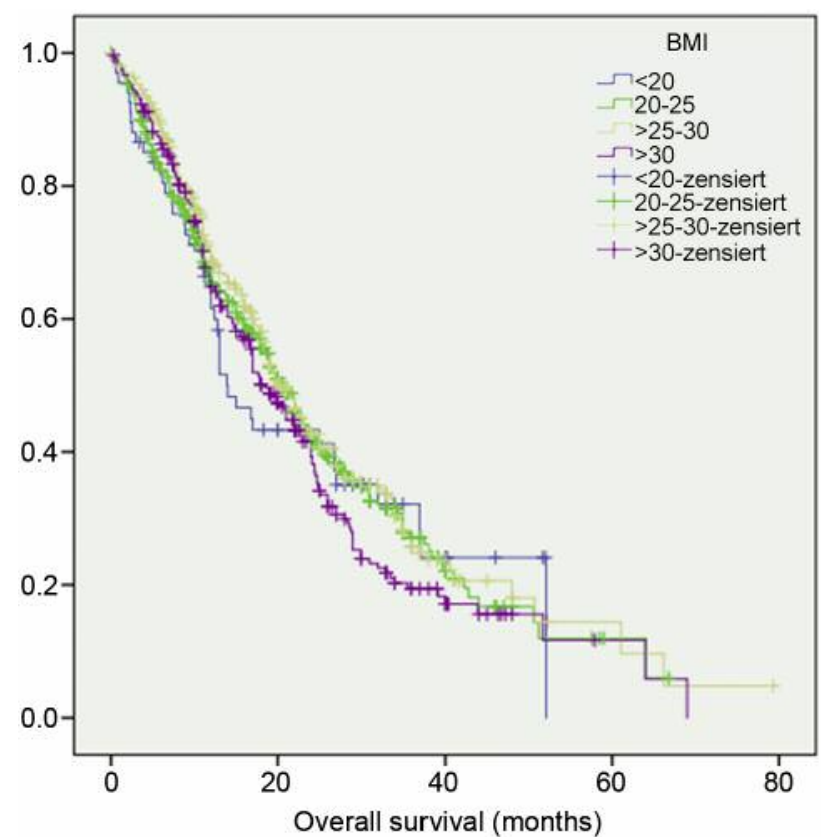

Figure 2. Overall survival regarding various body mass index (BMI) values, $p=0.386$.

Malnutrition and underweight in ovarian cancer patients are associated with worse survival. Recently, a prospective study showed poor overall survival in patients with low BMI before primary cytoreductive surgery (15). According to our data, no differences in progression-free and overall survival exist regarding chemotherapy in analyzed trials.

Bandera et al. reported that obese ovarian cancer patients received lower doses in milligrams per kilogram of body weight due to dose adjustment, so-called average relative dose intensity (ARDI), in comparison to normal weighted women $(p<0.001)$ (6). That is due to different pharmacokinetics in obese patients (17) and also due to the fact that higher BMI significantly increased the risk of dose reduction so that overweight women received $38 \%$ and $45 \%$ lower doses of paclitaxel and carboplatin (6). At the same time overall and ovarian-specific mortality increased with an ARDI lower than $70 \%$ of the regular dose (6). Similar observations were made by Chambers et al. in obese patients with colorectal cancer (18). Chambers et al. and Carroll et al. (19) also reported that full doses of chemotherapy did not increase the incidence and severity of toxicities in obese patients. These findings suggest that chemotherapy dosing in obese patients should be examined and monitored more closely in prospective trials.

Severe hematological toxicity and higher medication intake are associated with higher BMI in ovarian cancer

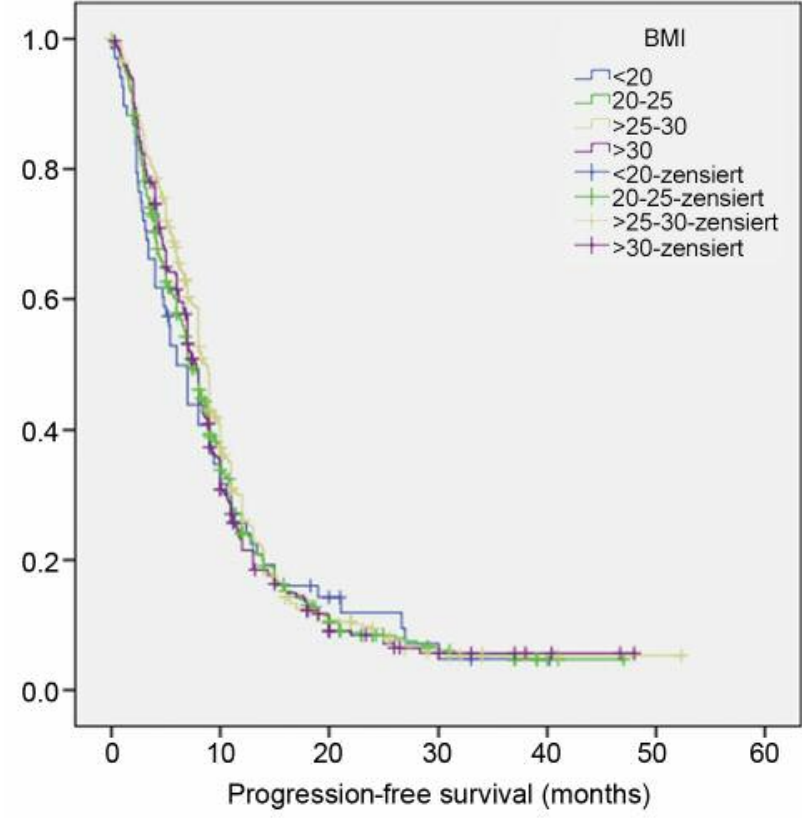

Figure 3. Progression-free survival regarding various body mass index (BMI) values, $p=0.488$.

patients. Moreover, fewer chemotherapy cycles and preterm discontinuation are more frequent in patients with lower BMI. Despite these differences, no influence on survival was observed.

Our study showed the significance of BMI regarding chemotherapy. This issue should be addressed in future trial planning processes. Clinical studies on the nutritional status, BMI optimization and therefore improvement of the quality of life are necessary.

\section{References}

1 Torre LA, Bray F, Siegel RL, Ferlay J, Lortet-Tieulent J and Jemal A: Cancer statistics, 2012. CA Cancer J Clin 65: 87-108, 2015.

2 Dixon SC, Nagle CM, Thrift AP, Pharoah PD, Pearce CL, Zheng W and Painter JN; AOCS Group \& Australian Cancer Study (Ovarian Cancer), Chenevix-Trench G, Fasching PA, Beckmann MW, Lambrechts D, Vergote I, Lambrechts S et al: Adult body mass index and risk of ovarian cancer by subtype: a Mendelian randomization study. Int J Epidemiol 45: 884-895, 2016.

3 Pergialiotis V, Doumouchtsis SK, Perrea D and Vlachos GD: The impact of underweight status on the prognosis of ovarian cancer patients: a meta-analysis. Nutr Cancer 68: 918-925, 2016.

4 Gutierrez F, Gonzalez-de-la-Fuente GA, Nazco GJ, Oramas J and Batista N: Hematological toxicity of carboplatin for gynecological cancer according to body mass index. Eur J Clin Pharmacol 72: 1083-1089, 2016. 
5 Duska LR, Java JJ, Cohn DE and Burger RA: Risk factors for readmission in patients with ovarian, fallopian tube and primary peritoneal carcinoma who are receiving front-line chemotherapy on a clinical trial (GOG 218): an NRG oncology/gynecologic oncology group study (ADS-1236). Gynecol Oncol 139: 221-227, 2015.

6 Bandera EV, Lee VS, Rodriguez-Rodriguez L, Powell CB and Kushi LH: Impact of chemotherapy dosing on ovarian cancer survival according to body mass index. JAMA Oncol 1: 737-745, 2015.

7 Au-Yeung G, Webb PM, DeFazio A, Fereday S, Bressel M and Mileshkin L: Impact of obesity on chemotherapy dosing for women with advanced stage serous ovarian cancer in the Australian ovarian cancer study (AOCS). Gynecol Oncol 133: 16-22, 2014.

8 Gordiner ME, Dizon DS, Fleming EL, Weitzen S, Schwartz J and Parker LP: Elevated body mass index does not increase the risk of palmar-plantar erythrodysesthesia in patients receiving pegylated liposomal doxorubicin. Gynecol Oncol 103: 72-74, 2006.

9 Tetsche MS, Dethlefsen C, Pedersen L, Sorensen HT and Norgaard M: The impact of comorbidity and stage on ovarian cancer mortality: a nationwide Danish cohort study, BMC Cancer 8: 31, 2008.

10 Woopen H, Richter R, Ismaeel F, Chekerov R, Roots I and Siepmann T: The influence of polypharmacy on grade III/IV toxicity, prior discontinuation of chemotherapy and overall survival in ovarian cancer. Gynecol Oncol 140: 554-558, 2016.

11 Woopen H, Richter R, Chekerov R, Siepmann T, Ismaeel F and Sehouli J: The influence of comorbidity and comedication on grade III/IV toxicity and prior discontinuation of chemotherapy in recurrent ovarian cancer patients. An individual participant data meta-analysis of the North-Eastern German Society of Gynecological Oncology (NOGGO). Gynecol Oncol 138: 735-740, 2015.

12 Sehouli J, Stengel D, Harter P, Kurzeder C, Belau A, Bogenrieder T, Markmann S, Mahner S, Mueller L, Lorenz R, Nugent A, Wilke J, Kuznik A, Doering G, Wischnik A, Sommer H, Meerpohl HG, Schroeder W, Lichtenegger W and OskayOezcelik G: Topotecan weekly versus conventional 5-day schedule in patients with platinum-resistant ovarian cancer: a randomized multicenter phase II trial of the North-Eastern German Society of Gynecological Oncology Ovarian Cancer Study Group. J Clin Oncol 29: 242-248, 2011.
13 Sehouli J, Stengel D, Oskay-Oezcelik G, Zeimet AG, Sommer H and Klare P: Nonplatinum topotecan combinations versus topotecan alone for recurrent ovarian cancer: results of a phase III study of the North-Eastern German Society of Gynecological Oncology Ovarian Cancer Study Group. J Clin Oncol 26: 3176-3182, 2008.

14 Sehouli J, Chekerov R, Reinthaller A, Richter R, GonzalesMartin A and Harter P: Topotecan plus Carboplatin versus standard therapy with paclitaxel plus carboplatin (PC) or gemcitabin plus carboplatin (GC) or pegylated doxorubicin plus carboplatin (PDLC): a randomized phase-III trial of the NOGGOAGO-Study Group-AGO Austria and GEICO-ENGOT-GCIG Intergroup study (HECTOR). Ann Oncol 27: 2236-2241, 2016.

15 Sehouli J, Ali P, Braicu EI, Chekerov R and Grabowski JP: The impact of preoperative malnutrition on surgery outcome and overall survival in ovarian or peritoneal cancer patients: A prospective study. J Clin Oncol 34: 5574, 2016.

16 Dahlberg SE, Schiller JH, Bonomi PB, Sandler AB, Brahmer JR and Ramalingam SS: Body mass index and its association with clinical outcomes for advanced non-small cell lung cancer patients enrolled on Eastern Cooperative Oncology Group clinical trials. J Thorac Oncol 8: 1121-1127, 2013.

17 Powis G, Reece P, Anmann DL and Ingle JW: Effect of body weight on the pharmacokinetics of cyclophosphamide in breast cancer patients. Cancer Chemother Pharmacol 20: 219-222, 1987.

18 Chambers P, Daniels SH, Thompson LC and Stephens RJ: Chemotherapy dose reductions in obese patients with colorectal cancer. Ann Oncol 23: 748-753, 2012.

19 Carrol JP, Protani MM, Nguyen L, Cheng ME, Fay M, Saleem M, Pillay PS, Walpole E and Martin JH: Toxicity and tolerability of adjuvant breast cancer chemotherapy in obese women. Med Oncol 31: 881, 2014.
Received August 19, 2018

Revised September 1, 2018

Accepted September 4, 2018 